\title{
Unilateral Optic Neuritis and Central Retinal Vasculitis due to Ocular Syphilis
}

Abbreviated Title: Syphilitic Optic Neuritis/CRA Vasculitis

\author{
Murtaza S. Khan, MD ${ }^{1}$ \\ Dulanji K. Kuruppu, MD ${ }^{1}$ \\ Tanav A. Popli, BA ${ }^{4}$ \\ Ramana S. Moorthy, MD $^{3}$ \\ Devin D. Mackay, MD ${ }^{1,2,3}$
}

\begin{abstract}
Affiliations:
From the Departments of Neurology ${ }^{1}$, Ophthalmology ${ }^{2}$, and Neurosurgery ${ }^{3}$ Indiana University, Indianapolis, Indiana and the Indiana University School of Medicine ${ }^{4}$, Indianapolis, Indiana
\end{abstract}

\section{Corresponding Author:}

Devin D. Mackay, M.D.

355 W. $16^{\text {th }}$ Street

Indianapolis, IN 46202

ddmackay@iupui.edu

\section{Disclosures:}

None of the authors has any financial or other conflicts of interest to disclose or any relevant proprietary interests.

Key Words: Central retinal artery occlusion, optic neuritis, steroids, syphilis, vasculitis

This is the author's manuscript of the article published in final edited form as:

Khan, M. S., Kuruppu, D. K., Popli, T. A., Moorthy, R. S., \& Mackay, D. D. (2020). Unilateral Optic Neuritis and Central Retinal Vasculitis due to Ocular Syphilis. Retinal Cases \& Brief Reports, 14(1), 35-38. https://doi.org/10.1097/ICB.0000000000000614 


\begin{abstract}
Purpose: Report a case of concurrent unilateral optic neuritis and central retinal artery occlusion as the presenting signs of syphilis.

Methods: A case report of a 22-year-old man with progressive unilateral vision loss.

Results: With no known prior history of syphilis, genital lesions, or other extraocular manifestations, the patient presented with pain with eye movements and decreased color vision. His vision dramatically worsened after a course of oral steroids. Examination was remarkable for severe right optic disc edema with a macular cherry red spot, and minimal posterior uveitis. MRI of the orbits with contrast revealed enhancement and enlargement of the distal right optic nerve. Fluorescein angiography demonstrated delayed filling of the right central retinal artery, suggestive of impending central retinal artery occlusion. Syphilis serologies were positive from the serum and CSF VDRL was reactive, consistent with neurosyphilis. Oral steroids were discontinued and vision improved with two weeks of intravenous penicillin.
\end{abstract}

Conclusion: This unusual case highlights one of the possible initial presentations of syphilis: unilateral optic neuritis and central retinal artery vasculitis with only minimal posterior uveitis. The worsening of vision after administration of oral steroids also highlights a potential complication of oral steroid use in the absence of a known etiology of vision loss. A thorough history and examination may be helpful in identifying risk factors for infectious causes, including syphilis, and should prompt additional evaluation.

\title{
Summary:
}

A 22-year-old man with pain with eye movements had worsening vision after oral steroid treatment. He had severe right optic disc edema with a macular cherry red spot. Testing confirmed neurosyphilis. This unusual case highlights unilateral optic neuritis and central retinal artery vasculitis as a rare initial presentation of syphilis.

\section{Introduction:}


The etiology of unilateral optic neuritis and retinal vasculitis includes primarily inflammatory and vascular causes. Here, we present a case of a young man with unilateral optic neuritis and retinal vasculitis involving the central retinal artery who experienced a significant worsening of vision after being treated with oral steroids, and further workup revealed positive cerebrospinal fluid and serum testing for syphilis. Concurrent unilateral optic neuritis and central retinal artery occlusion from infectious vasculitis is an extremely rare presentation of ocular syphilis. While rare, this case emphasizes the need for appropriate testing to screen for an infectious cause of optic neuritis and retinal vasculitis prior to consideration of any immunomodulatory therapies.

\section{Case Presentation}

A 22 year-old healthy Caucasian man presented with four weeks of right eye pain/retrobulbar pressure and two weeks of decreased color vision in the right eye. Two weeks prior to presentation, he saw an ophthalmologist and was found to have visual acuities of 20/25 in the right eye and 20/20 in the left eye. Fundoscopic exam revealed right optic disc edema and macular edema, for which he was prescribed an oral steroid taper. MRI of the orbits with and without contrast showed distal right optic nerve enhancement and enlargement (figure 1), consistent with anterior optic neuritis. Follow-up eleven days later revealed right eye visual acuity of 20/20, a right relative afferent pupillary defect, decreased macular edema, and temporal depression in the right eye on Humphrey visual fields. The following day, the patient awoke with drastically decreased vision in the right eye and worsening pain with eye movement. Examination revealed visual acuity of light perception only in the right eye and a large right relative afferent pupillary defect. He was restarted on oral prednisone and azithromycin, and referred to neuroophthalmology.

On presentation to the neuro-ophthalmology clinic 4 weeks after symptom onset, visual acuity was hand motion at 6 feet in the right eye, and 20/15 in the left eye. There was a $>1.8 \log$ unit right relative afferent pupillary defect and a sensory exotropia. Fundoscopic exam revealed severe right optic disc 
edema with complete obscuration of all major optic disc vessels and peripapillary flame-shaped hemorrhages that extended into the macula (figure 2). There was a macular cherry red spot (figure 2) in the right eye with surrounding pallor, consistent with central retinal artery insufficiency or occlusion.

Infectious workup revealed RPR was reactive with a titer of 1:128 and treponemal IgG was positive at > 8.0, and steroids were discontinued. Fluorescein angiography showed evidence of retinal vasculitis in the right eye with slow flow through the central retinal artery, but without complete occlusion (figure 3) and no evidence of late staining from chorioretinitis. The fundus autofluorescence photographs showed hyperautofluorescence of much of the peripapillary retina and posterior pole (figure 4). On hospital admission, a lumbar puncture showed cerebrospinal fluid (CSF) with a pleocytosis of 23 white blood cells/ $\mu \mathrm{L}$ (67\% lymphocytes) with normal glucose and a mildly elevated protein of $49 \mathrm{mg} / \mathrm{dL}$. VDRL CSF testing was positive with a titer of 1:4. Serum HIV testing was negative. On further questioning, the patient revealed a history of unprotected sex with men, but no prior diagnosis of syphilis or history of genital lesions. He was treated with 2 weeks of intravenous penicillin G. Three months after treatment, the RPR titer was 1:4, suggesting an adequate response to therapy. Follow-up examination after four months revealed a visual acuity of 20/125 with pinhole in the right eye, and pallor of the right optic disc . The retinal whitening had resolved and there was significant vascular attenuation in the right eye.

\section{Discussion}

Like the systemic manifestations of syphilis, ocular syphilis may present with a variety of manifestations, giving syphilis its moniker of "the great imitator." The most common presentation of ocular syphilis is panuveitis ${ }^{1}$, but other reported manifestations include keratitis, iritis, optic neuritis, macular edema, vitritis, and retinitis. Ocular involvement may occur at any stage of syphilis and can mimic other infections or even systemic inflammatory diseases, such as sarcoidosis. ${ }^{2}$ Central retinal artery occlusion from infectious vasculitis is an extremely rare presentation of syphilitic uveitis. The differential diagnoses of optic neuritis with central retinal artery territory hypoperfusion should include primarily 
autoimmune and infectious causes. The mechanism by which syphilis causes swelling of the optic nerve and central retinal artery vascular insufficiency has been partly elucidated. Approximately 3-4 weeks after infection, spirochetes are disseminated in the blood, and may affect multiple organ systems, including the central nervous system and blood vessels. ${ }^{3}$ In the secondary stage of syphilis infection, there is likely increased activation of B cells and plasma cells to fight the infection. As the disease becomes more disseminated and approaches late infection, there is an accumulated immune response with lymphocytic infiltration of blood vessels and tissues that can lead to a destructive endoarteritis. ${ }^{4}$ Syphilitic vasculitis in small vessels (Nissl-Alzheimer arteritis) is characterized by proliferation of endothelium and adventitia, and can lead to retinal and optic nerve ischemia when the central retinal artery or its branches, or the optic nerve blood supply (posterior ciliary arteries, central retinal artery branches, or pial vessel perforators) become involved. Alternatively, optic neuritis may result from inflammation due to spirochete involvement of the optic nerve head. ${ }^{5}$ We suspect that localized vasculitis of the central retinal artery contributed to the apparent cherry-red spot appearance in our case.

Optic neuritis is commonly an autoimmune process, and steroids are often used as treatment. However, atypical features, such as retinal abnormalities or a progressive clinical course, and risk factors for infection revealed by a thorough history and examination should prompt additional workup. There is no role for intermediate dose oral steroids $(\sim 1 \mathrm{mg} / \mathrm{kg} / \mathrm{d})$ in the management of even typical optic neuritis, as demonstrated by The Optic Neuritis Treatment Trial. Furthermore, systemic, periocular, or intraocular corticosteroids should never be used alone in the treatment of uveitis suspected of being infectious. Because steroid treatment of infectious etiologies may temporarily decrease inflammation and improve symptoms, but exacerbate the progression of disease ${ }^{6}$, careful consideration must first be given to ruling out potential infectious causes of ocular inflammatory disease. Steroids should be avoided in ocular syphilis, except perhaps as a brief adjunctive treatment in the setting of a Jarisch-Herxheimer reaction, in which bacteriolysis due to treatment causes an exaggerated host response, with the potential to further 
damage host tissues. This case highlights an unusual, but treatable, cause of unilateral optic neuritis and central retinal artery vasculitis.

\section{References}

1. Mathew RG, Goh BT, Westcott MC. British Ocular Syphilis Study (BOSS): 2-year national surveillance study of intraocular inflammation secondary to ocular syphilis. Invest Ophthalmol Vis Sci 2014;55(8):5394-5400.

2. Rescigno RJ, Glatman M, Patel SN. A Complicated Case of Sarcoidosis. JAMA Ophthalmol 2014;132(5):649-650.

3. Baughn RE, Musher DM. Secondary Syphilitic Lesions. Clin Microbiol Rev 2005;18(1):205-216.

4. Carlson JA, Dabiri G, Cribier B, Sell S. The immunopathobiology of syphilis:the manifestations and course of syphilis are determined by the level of delayed-type hypersensitivity. Am J Dermatopathol 2011;33(5):433-460.

5. Pless ML, Kroshinsky D, LaRocque RC, et al. Case records of the Massachusetts General Hospital. Case 26-2010. A 54 year-old man with loss of vision and a rash. N Engl J Med 2010;363(9):865-874. 6. Eagling EM, Sanders MD, Miller SJH. Ischemic papillopathy. Clinical and fluorescein angiographic review of forty cases. Brit J Ophthal 1974;58:990-1008. 


\section{Figure Legends:}

Figure 1. MRI of the orbits, T1 axial post-contrast images.

Enhancement and enlargement of the distal portion of the right optic nerve (arrow) and posterior globe.

Figure 2. Severe right optic disc edema with macular cherry red spot.

Color fundus photograph showing severe right optic disc edema with complete obscuration of all optic disc vessels. Peripapillary flame-shaped hemorrhages are present, and extend into the macula. The macular cherry red spot seen here (arrow) is consistent with central retinal artery territory hypoperfusion found on fluorescein angiography.

Figure 3. Fluorescein Angiography, right eye.

Early phase (1 minute) on left shows delayed venous filling in the laminar venous phase due to profound delay in circulation time with some arteriolar reperfusion. Late phase (5 minutes) on right shows staining of optic nerve head and proximal perivascular area.

Figure 4. Fundus autofluorescence photograph.

Hypoautofluorescent edematous right optic disc and hyperautofluorescent posterior pole from probable retinal ischemia or local inflammation. 